\title{
Optimal Tilings and Best Basis Search in Large Dictionaries.
}

\author{
Yan Huang
}

Ilya Pollak

Abstract-We develop a new framework of multitree dictionaries which include many previously proposed dictionaries as special cases. We show how to efficiently find the best object in a multitree dictionary using a recursive dynamic programming algorithm. We apply our framework to find the best rectangular tiling of an image domain.

\section{INTRODUCTION.}

Over the last ten years, a number of research efforts have concentrated on developing adaptive algorithms for representing and approximating signals in overcomplete dictionaries. Some examples are best basis search in dyadic wavelet packet trees and dyadic local cosine trees [3], matching pursuit [14] and its variants, and basis pursuit [2]. The applications of such algorithms include compression [18], extraction of time-frequency features [4], [6], [20] and geometric features [10], noise removal [11], [12], [16], [17], and others. The ultimate objective of these efforts is to adaptively compute a parsimonious representation at a low computational cost.

Among the aforementioned methods, the best basis search algorithms have the lowest complexity. The original paper on best basis search [3] considered the wavelet packet bases and bases of local cosines on dyadic intervals. In each of these two cases, all the bases in the dictionary can be organized using a single tree: a dyadic tree in 1-D and a quadtree in 2-D. This organization was exploited in [3] to devise a fast dynamic programming algorithm to find the best basis for any additive cost function.

Since then, a number of efforts have sought to lift the restrictions that a fixed dyadic/quadtree structure imposes on the underlying dictionary. Search methods for various dictionaries that correspond to different sets of possible timefrequency tilings have been proposed, such as the double-tree algorithm [7], time-frequency trees [19], [21], space-frequency trees [8], adaptive Haar-Walsh tilings [13], anisotropic wavelet packets [1], [5], anisotropic cosine packets [1], and mixed isotropic/anisotropic packets [1].

The main contribution of the present paper is a new framework of multitree dictionaries which include many previously proposed dictionaries as special cases. We show how to efficiently find the best object in a multitree dictionary using

This work was supported in part by a National Science Foundation CAREER award CCR-0093105, a Purdue Research Foundation grant, and an NSF CAREER award CCR-0237633.

Y. Huang, I. Pollak, and C.A. Bouman are with the School of Electrical and Computer Engineering, Purdue University, 1285 EE Building, West Lafayette, IN 47907. Corresponding author's e-mail: ipollak@ecn.purdue.edu. M.N. Do is with the Department of Electrical and Computer Engineering and Beckman Institute, University of Illinois at Urbana-Champaign.

\author{
Minh N. Do
}

Charles A. Bouman

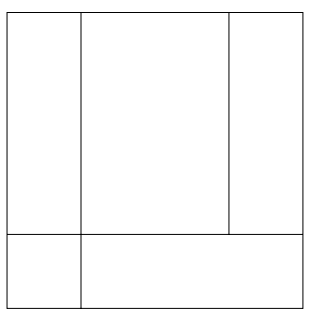

(a) An acceptable tiling.

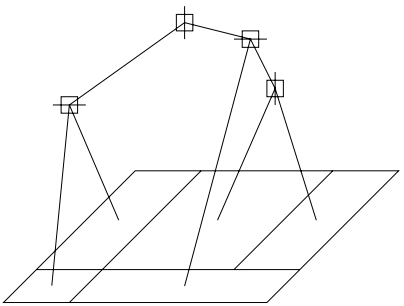

(c) A sequence of splits.

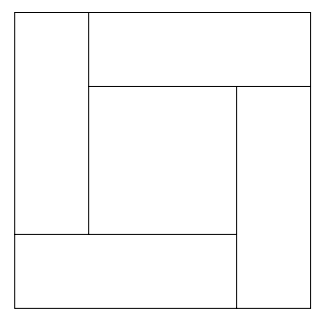

(b) An unacceptable tiling.

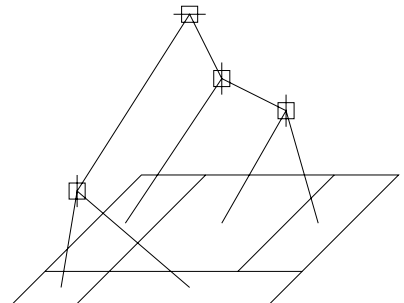

(d) Another sequence of splits.
Fig. 1. An illustration of tilings and sequences of splits. (a) An acceptable tiling-i.e., a tiling that can be obtained via a sequence of binary splits. (b) An unacceptable tiling. (c) A sequence of splits that leads to the tiling in (a). (d) Another sequence of splits that leads to the tiling in (a).

a recursive dynamic programming algorithm. This is done in Section III. We lead into that discussion with a specific example, namely, optimal rectangular tiling algorithms which are presented in Section II. We argue in Section III that the algorithms of Sections II are special cases of a general dynamic programming algorithm for finding the best object in a multitree dictionary. We moreover show in Section IV that another special case is a recursive version of the algorithm from [1], [5] to find the best 2-D anisotropic wavelet packet basis. Yet another special case of our algorithm is the best multitree local cosine basis [9].

\section{Optimal Rectangular Tilings.}

We consider all images supported on a rectangular domain $Q \subset \mathbb{Z}^{2}$. Suppose we are given an image $f$ and would like to segment it into rectangular regions $P_{1}, P_{2}, \ldots, P_{d}$ so as to minimize a cost which is equal to the sum of the costs of the individual rectangles:

$$
\sum_{i=1}^{d} e\left(P_{i}\right),
$$

where $e$ is a cost function which is application specific.

We restrict our choice of tilings, and only consider those tilings that can be obtained by a recursive binary splitting process: 
- start with a tiling which consists of a single rectanglenamely, the whole image domain;

- for every rectangle in the tiling,

either keep it and do not split it ever again, or split it into two rectangles;

- continue until all the rectangles in the tiling are labeled "never split again".

A rectangular tiling which can be obtained through this procedure is called an acceptable tiling. We will use $\mathcal{A}$ to denote the set of all acceptable tilings of the image domain $Q$. An acceptable tiling is illustrated in Fig. 1(a). The rectangular tiling depicted in Fig. 1(b) cannot be obtained through the binary splitting process described above, even though every region in the tiling is a rectangle. This tiling is therefore not an acceptable tiling.

The binary splitting process is conveniently visualized as a tree, with every node of the tree corresponding to a rectangular region of the image, as shown in Fig. 1(c). In our diagrams, we draw a vertical line through a node in the tree if the corresponding region is split along a vertical line, and a horizontal line if the corresponding region is split along a horizontal line. The yield ${ }^{1}$ of the binary tree is then the tiling of the image domain. The set of all such trees will give us the set of all acceptable tilings (however, several different trees may correspond to the same tiling, as shown in Fig. 1(c,d)). Our optimal tiling problem is therefore equivalent to searching for a tree with the least costly yield.

We now show that this problem can be solved efficiently, using a polynomial-complexity algorithm. Let $C_{P}^{*}$ be the optimal cost for a rectangle $P$. Our algorithm starts from the entire image domain $Q$, and makes the following recursive call:

$$
C_{P}^{*}=\min \left\{e(P), \min \left\{C_{P^{\prime}}^{*}+C_{P^{\prime \prime}}^{*}\right\}\right\},
$$

where the inner minimization is done over all ordered pairs of rectangles $\left(P^{\prime}, P^{\prime \prime}\right)$ which partition the rectangle $P$ :

$$
P=P^{\prime} \cup P^{\prime \prime} \text { and } P^{\prime} \cap P^{\prime \prime}=\varnothing .
$$

We always assume that, if the split it horizontal, then $P^{\prime}$ is on top of $P^{\prime \prime}$, and if the split is vertical, then $P^{\prime}$ is to the left of $P^{\prime \prime}$.

The recursive call (1) terminates at the pixels:

$$
\text { if } P \text { is a pixel, then } C_{P}^{*}=e(P) \text {. }
$$

To avoid repetitive calculation, we store the optimal cost and the optimal split for each rectangle in a global table. Before making a recursive call for any rectangle $P$, the table is consulted to make sure that $P$ has not been visited before. If the original image domain is $N_{1} \times N_{2}$, it has $O\left(N_{1}^{2} N_{2}^{2}\right)$ different subrectangles, and therefore maintaining the table requires $O\left(N_{1}^{2} N_{2}^{2}\right)$ memory. With this table, we only need to make one recursive call per rectangle. Since each recursive call involves $O\left(N_{1}+N_{2}\right)$ comparisons to calculate $C_{P}^{*}$ via Eq. (1), the computational complexity of the search algorithm is $O\left(N_{1}^{2} N_{2}^{2}\left(N_{1}+N_{2}\right)\right)$ which is $O\left(N^{2.5}\right)$ for a square image with $N$ pixels, $N_{1}=N_{2}=\sqrt{N}$.

\footnotetext{
${ }^{1}$ The yield of a tree is the set of all leaves of the tree.
}

The preceding discussion supposes that the individual costs $e(P)$ have been precomputed for every rectangle $P$. We analyze this computation using the following simple cost:

$$
e(P)=\sum_{\left(n_{1}, n_{2}\right) \in P}\left(f\left(n_{1}, n_{2}\right)-f_{P}\right)^{2}+c,
$$

which results in the following overall segmentation cost:

$$
\sum_{i=1}^{d} \sum_{\left(n_{1}, n_{2}\right) \in P_{i}}\left(f\left(n_{1}, n_{2}\right)-f_{P_{i}}\right)^{2}+c d
$$

where

$f\left(n_{1}, n_{2}\right)$ is the pixel value at the location $\left(n_{1}, n_{2}\right)$;

$f_{P_{i}}$ is the average of the image $f$ over the rectangle $P_{i}$;

$d$ is the number of rectangles in the segmentation;

$c$ is a weighting parameter.

The first term in this cost penalizes excessive variability of the image within each rectangle of the tiling. If the second term did not exist-i.e., if we had $c=0$-then a best solution would be to segment the image domain into $1 \times 1$ rectangles. The second term penalizes the number of rectangles in the tiling.

For this particular cost function (3), computing $e(P)$ for every rectangle $P$ can be done very efficiently by defining the following two statistics:

$$
\begin{aligned}
& \rho_{1}(f, P)=\sum_{\left(n_{1}, n_{2}\right) \in P} f\left(n_{1}, n_{2}\right)=|P| f_{P} \\
& \rho_{2}(f, P)=\sum_{\left(n_{1}, n_{2}\right) \in P} f\left(n_{1}, n_{2}\right)^{2},
\end{aligned}
$$

and noticing that, if we know these two statistics for a pair of rectangles $\left(P^{\prime}, P^{\prime \prime}\right)$ which partition a rectangle $P$, we can calculate $e(P)$ in $O(1)$ time as follows:

$$
\begin{aligned}
\rho_{1}(f, P) & =\rho_{1}\left(f, P^{\prime}\right)+\rho_{1}\left(f, P^{\prime \prime}\right) \\
\rho_{2}(f, P) & =\rho_{2}\left(f, P^{\prime}\right)+\rho_{2}\left(f, P^{\prime \prime}\right) \\
e(P) & =\rho_{2}(f, P)-\rho_{1}^{2}(f, P) /|P|+c .
\end{aligned}
$$

This is used to compute all the costs in a bottom-up fashion, with both time and space complexity $O\left(N_{1}^{2} N_{2}^{2}\right)$.

The overall time complexity of the algorithm-i.e., the computation of the costs and the recursive search combinedis $O\left(N_{1}^{2} N_{2}^{2}\left(N_{1}+N_{2}\right)\right)$. The overall space complexity is $O\left(N_{1}^{2} N_{2}^{2}\right)$. In some situations, the computation of the costs may be more complex and in fact may dominate the computational complexity of the overall algorithm.

Note that reducing the number of acceptable rectangular tilings may result in a lower computational complexity of the algorithm. For example, we can restrict the search space if we only allow a rectangle to be split into two congruent rectangles, as was done in, e.g., [5]. In other words, we can impose that during our recursive binary splitting process, an $n_{1} \times n_{2}$ rectangle may only be split either into two $n_{1} / 2 \times n_{2}$ rectangles, or into two $n_{1} \times n_{2} / 2$ rectangles. This scenario is similar to the anisotropic wavelet packets [1], [5]. ${ }^{2}$ It can

\footnotetext{
${ }^{2}$ The scenario which is similar to the classical wavelet packets results from imposing that, furthermore, any horizontal split must be followed by a vertical one, and vice versa. In other words, if an $n_{1} \times n_{2}$ rectangle resulted from a horizontal split, it is only allowed to be split into two $n_{1} \times n_{2} / 2$ rectangles; and if it resulted from a vertical split, it is only allowed to be split into two $n_{1} / 2 \times n_{2}$ rectangles.
} 


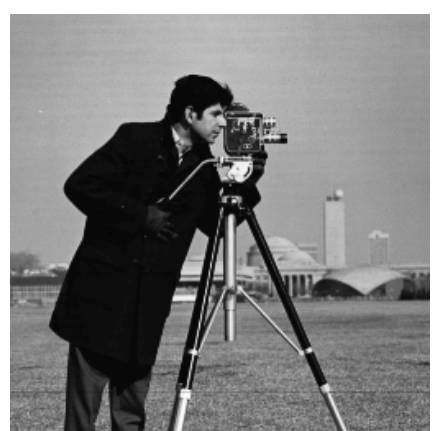

(a) Cameraman image.

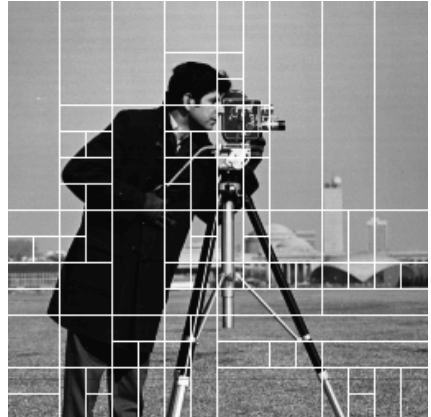

(b) Best dyadic tiling, cost 0.565

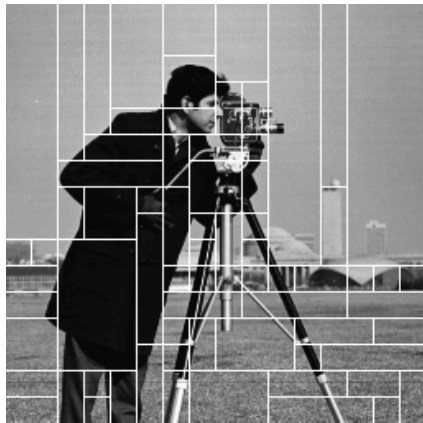

(c) Best arbitrary tiling, cost 0.441
Fig. 2. A $256 \times 256$ cameraman image and its best rectangular tilings with the smallest block size $16 \times 16$ : (b) best dyadic tiling, cost 0.565 ; (c) best arbitrary tiling, cost 0.441 .

be shown that in this case, the total number of possible rectangular tiles is $O\left(N_{1} N_{2}\right)$, and therefore the computation of the costs has time and space complexity $O\left(N_{1} N_{2}\right)$. The minimization in Eq. (1) is $O(1)$ since it now involves choosing one of no more than three options: horizontal split or vertical split or no split. Therefore, both the time and space complexity of the search is $O\left(N_{1} N_{2}\right)$, which is also the overall complexity of the algorithm-i.e., the computation of the costs and the recursive search combined. In this case, the complexity is linear in the number of pixels.

Our rectangular tiling algorithms are illustrated in Fig. 2.

\section{Multitree Dictionaries.}

The rectangular tiling process described above is a splitting process which recursively builds an ordered collection of objects, starting with a single object. A tree is constructed whose yield (i.e., the set of leaves) is a representation of the data. Such processes are conveniently described using the formalism of grammars. We define a grammar $G=(A, S)$ to be a pair of two sets:

- a set $A$ of atomic objects, and

- a set $S$ of allowed splits (also called productions) of the form $a \rightarrow \alpha$ where $a \in A$, and $\alpha$ is a finite sequence of elements of $A$.

Depending on the application, the elements of $A$ can be orthogonal bases, rectangular tiles, segments of the time axis, or something else. By starting with a single element of $A$, we can generate various sequences of elements of $A$ via recursive splitting. This process can be visualized as a tree where each split $a \rightarrow \alpha$ is depicted as a node labeled $a$ whose children are labeled with the elements of $\alpha$, left to right. We let $T(G)$ be the set of all trees that can be produced ${ }^{3}$ by the grammar $G$. For each tree $t \in T(G)$, we let the yield $Y(t)$ of $t$ be the unordered set of the labels of the leaves of $t$.

Note that in the rectangular tiling example, the splitting process was binary and led to dyadic trees. Here, we allow splits into an arbitrary finite number of atomic objects.

We let $T_{a}(G)$ be the set of all trees in $T(G)$ whose root is labeled $a$. We say that a grammar $G=(A, S)$ is finite-depth if, for every $a \in A, T_{a}(G)$ is a finite set.

For any finite-depth grammar $G=(A, S)$ and any $a \in A$, a multitree dictionary $\mathcal{D}_{a}(G)$ is the collection of yields of all the trees in $T_{a}(G)$ :

$$
\mathcal{D}_{a}(G)=\left\{\mathcal{B} \mid \mathcal{B}=Y(t) \text { for some } t \in T_{a}(G)\right\} .
$$

Suppose that each atomic object $a^{\prime} \in A$ is assigned a cost $e\left(a^{\prime}\right)$, and that the cost $C(\mathcal{B})$ of any object $\mathcal{B} \in \mathcal{D}_{a}$ is the sum of the individual costs of all the atomic objects comprising $\mathcal{B}$ :

$$
C(\mathcal{B})=\sum_{a^{\prime} \in \mathcal{B}} e\left(a^{\prime}\right) .
$$

We would like to find the best object in the dictionary $\mathcal{D}_{a}$, i.e., the object $\mathcal{B}_{a}^{*}$ whose cost is the smallest:

$$
\mathcal{B}_{a}^{*}=\arg \min _{\mathcal{B} \in \mathcal{D}_{a}} C(\mathcal{B}) .
$$

We denote the corresponding cost by $C_{a}^{*}: C_{a}^{*}=C\left(\mathcal{B}_{a}^{*}\right)$. We let $S_{a}$ be the set of all allowed splits of a fixed atomic object $a$. To illustrate our fast recursive algorithm for best object search, we first suppose that $S_{a}=\{a \rightarrow b c\}$. There is a single tree in $T_{a}(G)$ which consists of one node labeled $a$, and whose yield is therefore $\{a\}$, with cost $C(\{a\})=e(a)$. For any other tree $t \in T_{a}(G)$, its left subtree $t_{\text {left }}$ is in $T_{b}(G)$, and its right subtree $t_{\text {right }}$ is in $T_{c}(G)$. Therefore, $Y(t)=$ $Y\left(t_{\text {left }}\right) \cup Y\left(t_{\text {right }}\right)$, and, since the cost is additive,

$$
C(Y(t))=C\left(Y\left(t_{\text {left }}\right)\right)+C\left(Y\left(t_{\text {right }}\right)\right) .
$$

Consequently, the optimal object is:

$$
\mathcal{B}_{a}^{*}= \begin{cases}\mathcal{B}_{b}^{*} \cup \mathcal{B}_{c}^{*} & \text { if } C_{b}^{*}+C_{c}^{*}<e(a) \\ \{a\} & \text { otherwise. }\end{cases}
$$

In other words, we find the best objects $\mathcal{B}_{b}^{*}$ and $\mathcal{B}_{c}^{*}$ in the dictionaries $\mathcal{D}_{b}$ and $\mathcal{D}_{c}$, respectively, and compare their total cost with the cost of $\{a\}$.

We have a similar recursion in the general case. We let $R(a)$ be the set of the right-hand sides of all the elements of $S_{a}$. Then the possible candidates for $\mathcal{B}_{a}^{*}$ are

$$
\begin{array}{rll}
\bigcup_{b \in \alpha} \mathcal{B}_{b}^{*}, & \text { with cost } & \sum_{b \in \alpha} C_{b}^{*}, \text { for any } \alpha \in R(a), \text { and } \\
\{a\}, & \text { with cost } & e(a) .
\end{array}
$$

Let

$$
\alpha_{a}^{*}=\arg \min _{\alpha \in R(a)} \sum_{b \in \alpha} C_{b}^{*}
$$

${ }^{3}$ We assume that our recursive tree generation process can stop after any number of recursions. This is different from standard treatments of grammars [15] where the stopping is handled via distinguishing between nonterminal objects which must always branch, and terminal objects which may never branch. 


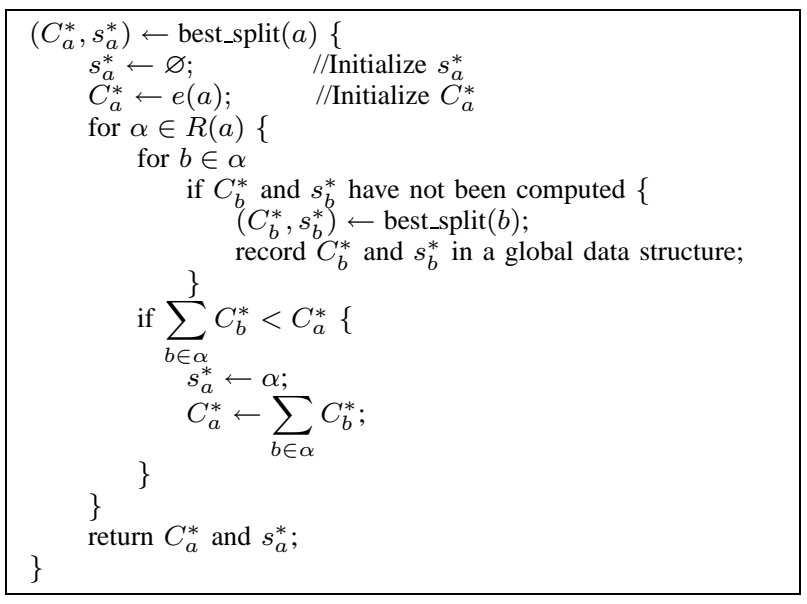

(a) Recursive calculation of the best splits and best costs.

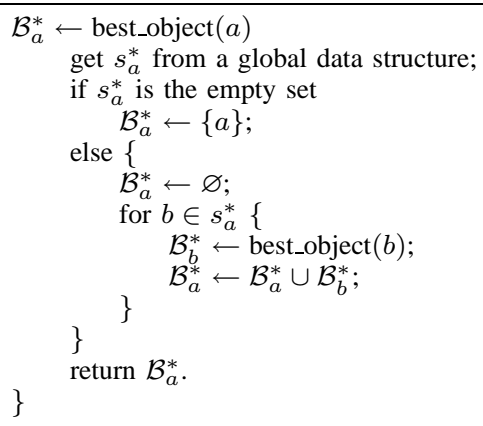

(b) Recursive generation of the best object.

Fig. 3. Pseudocode specification of a fast recursive algorithm for the bestobject search in a multitree dictionary: (a) the recursive calculation of the best costs and best splits; (b) the recursive generation of the best object.

Then

$$
\mathcal{B}_{a}^{*}= \begin{cases}\bigcup_{b \in \alpha_{a}^{*}} \mathcal{B}_{b}^{*} & \text { if } \sum_{b \in \alpha_{a}^{*}} C_{b}^{*}<e(a) \\ \{a\} & \text { otherwise. }\end{cases}
$$

The globally optimal search algorithm is a repeated, recursive use of Eqs. (5) and (6). A branch of the recursion terminates when $S_{b}=\varnothing$ : in this case, $\mathcal{B}_{b}^{*}=\{b\}$. The termination is guaranteed to happen in a finite number of steps for a finitedepth grammar. To avoid repetitive calculation, we store the optimal cost $C_{a}^{*}$ and the optimal split $s_{a}^{*}$ for each atomic object $a$ in a global table:

$$
\left(C_{a}^{*}, s_{a}^{*}\right)= \begin{cases}\left(\sum_{b \in \alpha_{a}^{*}} C_{b}^{*}, \alpha_{a}^{*}\right) & \text { if } \sum_{b \in \alpha_{a}^{*}} C_{b}^{*}<e(a) \\ (e(a), \varnothing) & \text { otherwise. }\end{cases}
$$

The pseudocode for the recursive calculation of $C_{a}^{*}$ and $s_{a}^{*}$ is given in Fig. 3(a). Once this recursive call is done, the best object can be generated from the global data structure using the pseudocode in Fig. 3(b).

The most significant computational burden is in computing and storing the best costs and best splits. To analyze this procedure, we let $A(a)$ be the union of $\{a\}$ and the set of all atomic objects which can be descendants of $a$. We let $S_{A(b)}$ be the set of all allowed splits of elements of $A(b)$. For each atomic object $b \in A(a)$, there is exactly one recursive call to the subroutine best_split of Fig. 3(a). During this call, the costs of all possible splits of $b$ are compared. The number of such comparisons is $\left|S_{b}\right|$. Therefore, the overall time complexity of the algorithm is $O\left(\left|S_{A(a)}\right|\right)$. As we have seen in the rectangular tiling example, however, this may be an overestimate: sometimes, the redundancy associated with searching over multiple trees which have the same yield may be eliminated leading to a lower time complexity.

The overall space complexity is $O(|A(a)|)$ since we need to store two numbers-the best cost and the best split-for each atomic object in $A(a)$. The key to controlling the time and space complexity is therefore keeping the sizes of the sets $S_{A(a)}$ and $A(a)$ low. In addition, as we have remarked before, the computation of the costs $e(a)$ could actually dominate the time complexity of the overall algorithm, and therefore another important guideline to a successful application of our algorithm is to use tractable cost functions.

\section{OTher ExAmples of Multitree Dictionaries.}

\section{A. Anisotropic Wavelet Packets.}

Recall that a single 1-D subband decomposition operation which is a basic building block of wavelet transforms and wavelet packet transforms takes any 1-D discrete sequence $u$ and transforms it into two other 1-D discrete sequences $u^{1}$ and $u^{2}$ via:

$$
u^{1}(n)=u * h(2 n) \quad \text { and } u^{2}(n)=u * g(2 n),
$$

where the lowpass filter $h$ and the highpass filter $g$ form a pair of conjugate mirror filters. When the sequence $u$ is viewed as a sequence of coefficients of a signal with respect to an orthogonal scaling basis $\mathcal{B}$, the sequences $u^{1}$ and $u^{2}$ can be interpreted as the sequences of coefficients of the same signal with respect to a coarser-scale orthogonal scaling basis $\mathcal{B}^{1}$ and a coarser-scale orthogonal wavelet basis $\mathcal{B}^{2}$, respectively.

The 1-D wavelet transform is obtained by recursively decomposing only the lowpass part-i.e., growing the tree on the left only. If we recursively decompose both parts, we grow a full 1-D wavelet packet tree. Two-dimensional wavelet packets alternate the decomposition (7) on the rows and the columns of an image, thereby producing a quadtree. The best basis search algorithm [3] is a bottom-up algorithm for pruning the full wavelet packet tree. An additive cost function is defined, and at every nonleaf node of the tree, a decision is made as to whether it is more costly to keep the children of the node or to prune them. Once the whole tree is traversed, the leaf nodes of the pruned tree correspond to the best basis.

A more flexible framework called anisotropic 2-D wavelet packets [1], [5], allows for any sequence of rowwise and columnwise applications of the decomposition operation (7), and is therefore a generalization of the classical wavelet packets.

Assuming that we consider $N_{1} \times N_{2}$ images where $N_{1}=$ $2^{L_{1}}$ and $N_{2}=2^{L_{2}}$, we define an atomic basis $\mathcal{B}_{i, j}^{p, q}$ for every scale $2^{i}$ along the rows, $0 \leq i \leq L_{1}$, for every scale $2^{j}$ along the columns, $0 \leq j \leq L_{2}$, and for every $p=0,1,2, \ldots, 2^{i}-1$ and $q=0,1,2, \ldots, 2^{j}-1$. These atomic bases are defined recursively as follows. 
- $\mathcal{B}_{0,0}^{0,0}$ is the finest-scale orthogonal scaling basis.

- For any $p, q, i, j, \mathcal{B}_{i+1, j}^{2 p, q}$ and $\mathcal{B}_{i+1, j}^{2 p+1, q}$ are the bases obtained via a columnwise application of the basic decomposition operation to $\mathcal{B}_{i, j}^{p, q}$.

- For any $p, q, i, j, \mathcal{B}_{i, j+1}^{p, 2 q}$ and $\mathcal{B}_{i, j+1}^{p, 2 q+1}$ are the bases obtained via a rowwise application of the basic decomposition operation to $\mathcal{B}_{i, j}^{p, q}$.

An anisotropic wavelet packet basis is defined to be any basis which can be obtained using the following recursive binary splitting process.

- Start with a representation $\mathcal{R}=\left\{\mathcal{B}_{0,0}^{0,0}\right\}$ consisting of a single basis $\mathcal{B}_{0,0}^{0,0}$.

- For each basis $\mathcal{B}_{i, j}^{p, q}$ in the current representation $\mathcal{R}$,

- either keep it and do not decompose ever again,

- or replace it with $\mathcal{B}_{i+1, j}^{2 p, q}$ and $\mathcal{B}_{i+1, j}^{2 p+1, q}$,

- or replace it with $\mathcal{B}_{i, j+1}^{p, 2 q}$ and $\mathcal{B}_{i, j+1}^{p, 2 q+1}$.

- Continue until all the bases in $\mathcal{R}$ are labeled "do not decompose ever again."

One full run of this binary splitting process can be visualized as a binary tree whose every node is associated with an atomic basis $\mathcal{B}_{i, j}^{p, q}$. The union of the atomic bases at the leaves of such a tree is called an anisotropic wavelet packet basis. A fast algorithm for finding the best anisotropic wavelet packet basis for an additive cost was provided in [1], [5]. It is easy to see that a specialization of our algorithm of Fig. 3 to the anisotropic wavelet packet dictionary is a restatement of the algorithm of [1], [5]. ${ }^{4}$

\section{B. Multitree Local Cosine Dictionaries.}

In [9], we propose an algorithm for best basis search in a dictionary of arbitrary local cosine bases on a discrete interval of length $N$. The problem is equivalent to finding the best segmentation of the interval into subintervals, and representing the data on each subinterval in the local cosine basis. This problem is therefore a 1-D version of our rectangular tiling problem, albeit with a very different cost.

\section{CONCLusions.}

We presented a general framework of multitree dictionaries and provided a recursive dynamic programming metaalgorithm for finding the best object in a multitree dictionary. We showed how to use our framework to find the best rectangular tiling, and demonstrated that several previously proposed dictionaries and best basis methods (such as anisotropic wavelet packets and multitree local cosine dictionaries) are special cases of our framework.

In the future we plan to further explore the flexibility of our framework and design various multitree dictionaries which allow a fast selection of the best representation in contexts such as time-frequency analysis, approximation, and compression.

\section{REFERENCES}

[1] N.N. Bennett. Fast algorithm for best anisotropic Walsh bases and relatives. J. of Appl. and Comput. Harmonic Analysis, 8:86-103, 2000.

[2] S. Chen, D.L. Donoho, and M.A. Saunders. Atomic decomposition by basis pursuit. SIAM Journal on Scientific Computing, 20(1):33-61, 1999.

[3] R.R. Coifman and M.V. Wickerhauser. Entropy based algorithms for best basis selection. IEEE Trans. Inf. Th., 38(2):713-718, March 1992.

[4] I. Cohen, S. Raz, and D. Malah. Orthonormal shift-invariant adaptive local trigonometric decomposition. Sig. Proc., 57(1):43-64, Feb. 1997.

[5] D. Donoho. CART and best-ortho-basis: A connection. Ann. Stat., 25:1870-1911, 1997.

[6] R. Gribonval, P. Depalle, X. Rodet, E. Bacry, and S. Mallat. Sound signals decomposition using a high resolution matching pursuit. In Proc. Computer Music Conf. (ICMC-96), pp. 293-296, Aug. 1996.

[7] C. Herley, J. Kovačević, K. Ramchandran, and M. Vetterli. Tilings of the time-frequency plane: construction of arbitrary orthogonal bases and fast tiling algorithms. IEEE Trans. Sig. Proc., 41(12):3341-3359, Dec. 1993.

[8] C. Herley, Z. Xiong, K. Ramchandran, and M.T. Orchard. Joint spacefrequency segmentation using balanced wavelet packet tree for least-cost image representation. IEEE Trans. Im. Proc., 6(9):1213-1230, Sep. 1997.

[9] Y. Huang, I. Pollak, C.A. Bouman, and M.N. Do. New algorithms for best local cosine basis search. Submitted to ICASSP-2004.

[10] S. Jaggi, W.C. Karl, S. Mallat, and A.S. Willsky. High resolution pursuit for feature extraction. J. of Appl. and Comput. Harmonic Analysis, 5:428$449,1998$.

[11] H. Krim and J.-C. Pesquet. On the statistics of best bases criteria. In Wavelets and Statistics, Lecture Notes in Statistics, A. Antoniadis, Ed., pp. 193-207. Springer-Verlag, 1995.

[12] H. Krim, D. Tucker, S. Mallat, D. Donoho. On denoising and best signal representation. IEEE Trans. Inf. Th., 45(7):2225-2238, Nov. 1999.

[13] M. Lindberg and L.F. Villemoes. Image compression with adaptive HaarWalsh tilings. In Wavelet Applications in Signal and Image Processing VIII, Proc. SPIE 4119, 2000.

[14] S.G. Mallat and Z. Zhang. Matching pursuits with time-frequency dictionaries. IEEE Trans. Sig. Proc., 41(12):3397-3415, Dec. 1993.

[15] C. Manning and H. Schütze. Foundations of Statistical Natural Language Processing. MIT Press, 1999.

[16] P. Moulin. Signal estimation using adapted tree-structured bases and the MDL principle. In Proc. IEEE-SP Int. Symp. TFTS, pp. 141-143, Paris, June 1996.

[17] J.-C. Pesquet, H. Krim, D. Leporini, and E. Hamman. Bayesian approach to best basis selection. In Proc. ICASSP-96 pp. 2634-2638, Atlanta, USA, May 1996.

[18] K. Ramchandran and M. Vetterli. Best wavelet packet bases in a ratedistortion sense. IEEE Trans. Im. Proc., 2(2):160-175, Apr. 1993.

[19] C.M. Thiele and L.F. Villemoes. A fast algorithm for adapted timefrequency tilings. J. of Appl. and Comput. Harmonic Analysis, 3:91-99, 1996.

[20] L.F. Villemoes. Adapted bases of time-frequency local cosines. Preprint, Feb. 2000, www. math.kth. se/ larsv/publ.html.

[21] Z. Xiong, K. Ramchandran, C. Herley, and M.T. Orchard. Flexible treestructured signal expansions using time-varying wavelet packets. IEEE Trans. Sig. Proc., 45(2):333-345, Feb. 1997.

\footnotetext{
${ }^{4} \mathrm{~A}$ minor difference is that the pseudocode of [1], [5] is bottom-up while our pseudocode is recursive.
} 